Tuberkulose 1951;8(suppl 1):13

\title{
Origine des malades
}

Durant la période d'observation qui est à la base de cette etude, la population du canton de Neuchâtel a subi une importante diminution. En 1921, elle était de 131738 habitants et en 1941 de 118 231. Quant à la mortalité par tuberculose, elle passait de 14,7 pour 10000 habitants en 1921 à 5,1 en 1941. En 1950, elle est de 3,0.

Un canton de Timportance de celui de Neuchâtel ne pouvait four-nir un contingent de tuberculeux suffisant pour remplir notre maison qui compte 100 lits pour adultes, pratiquement exclusivement reserves aux tuberculoses intrathoraciques. D'autre part, le canton de Neuchâtel est un des cantons suisses où la mortalité par tuberculose est la plus faible, où elle a le plus régressé. Pour cette double raison, les malades d'origine neuchâteloise ou domiciliés dans le canton n'ont jamais représenté, au maximum, que le 76\% (1927) et au minimum que le 54\% (1941) de $\Gamma$ effectif théorique total de 100 malades.

Les lits disponibles ont été occupés par des malades venant d'autres cantons, en particulier par des Fribourgeois qui, en 1941, ont atteint un maximum avec 30\% de l'effectif théorique total. Nous n'avons hébergé que très exceptionnellement des étrangers venant du dehors.

Les malades hommes neuchâtelois d'origine ou de domicile se recrutaient essentiellement parmi les horlogers, les ouvriers d'usine de moyenne ou de petite mécanique, les artisans et les employes de bureau. Ils rentrent done pour la grande majorité dans la catégorie des «metiers sédentaires»6, c'est-à-dire des metiers réclamant peu d'efforts physiques. Au contraire, les malades fribourgeois, pour la plupart, étaient des agriculteurs, des ouvriers agricoles ou des manoeuvres, par consequent des sujets appartenant à la catégorie des «metiers de force», soit «des metiers exigeant de sérieux efforts et s'exécutant quelquefois sous les intempéries»7. En gros, trois cin-quièmes de nos malades hommes appartenaient à la catégorie des «metiers sédentaires» et deux cinquièmes à celle des «métiers de force».

6 Jeanne Hummel: Les conceptions actuelles en matière de «post-cure» des tuberculeux. Rev. suisse d'hygiène 1949, fasc. 9.

7 Jeanne Hummel: op. cit. 